\title{
Long Term Follow-Up After Atrioventricular Node Ablation and Right Ventricular Pacing: Effects on Clinical Events and Left Ventricular Function
}

\author{
Isabelle Nault ${ }^{*}$, Gilles O’Hara, François Philippon, Éric Larose, Franck Molin, Louis Blier, \\ Marcel Gilbert and Jean Champagne
}

2725 Chemin Ste-Foy, Québec (QC), G1V 4G5, Canada

\begin{abstract}
Among rate control strategies for atrial fibrillation is atrioventricular junction ablation with ventricular pacing. Concerns about the long term safety of this procedure emerge as studies report deleterious effects of right ventricular pacing. A cohort of patients having undergone atrioventricular node ablation and right ventricular pacing was retrospectively studied. After 5 years of follow-up, a small decrease in left ventricular ejection fraction was observed and $19 \%$ of patients suffered at least one episode of decompensated heart failure, often in association with contributing cardiovascular comorbidities. Atrioventricular node ablation with pacing can still safely be used in selected patients to control rate in atrial fibrillation.
\end{abstract}

Keywords: Atrial fibrillation, atrioventricular junction ablation, catheter ablation, right ventricular pacing.

\section{BACKGROUND}

Atrial fibrillation (AF) affects $1 \%$ of the general population, with prevalence increasing with age. Management strategies have evolved in the past years, with publication of landmarks studies like the AFFIRM trial [1] that showed the equivalence of rate and rhythm control strategies on total mortality. Evolution in catheter ablation techniques for $\mathrm{AF}$ also greatly influenced the way physicians treat $\mathrm{AF}$, and freedom from atrial fibrillation is achieved in more than 75 $\%$ of patients following $\mathrm{AF}$ ablation [2]. Atrioventricular (AV) node ablation and pacing, mostly achieved from the right ventricular apex, (ablate and pace strategy) has been one way to manage uncontrolled rapid atrial fibrillation. Although it is a palliative strategy, because patients usually remained in $\mathrm{AF}$, it has shown good results in improving symptoms and quality of life [3] with no adverse effect on survival $[4,5]$. Others have reported increased mortality if baseline left ventricular systolic function was altered [5] or following pacing at low heart rate in patients with previous rapid AF [6]. The ablate and pace strategy is now less commonly used because there is now ways to cure AF using percutaneous techniques, but also in fear of deleterious effects of pacing on left ventricular function [7-9]. Nevertheless, some patients who are poor candidate to primary atrial fibrillation ablation, for example high risk patients with multiple comorbidities, elderly patients, or some who simply refuse the risks inherent to the procedure can still be controlled with $\mathrm{AV}$ node ablation and pacing.

\section{OBJECTIVE}

This study was conducted to assess the long term evolution of patients following ablate and pace strategy in order to

\footnotetext{
*Address correspondence to this author at the 2725 Chemin Ste-Foy, Québec (QC), G1V 4G5, Canada; Tel: (418) 656-8711; Fax: (418) 656-4581; E-mail: isabellenault@gmail.com
}

evaluate its long term effects on left ventricular systolic function and adverse clinical events.

\section{METHOD}

This is a single center retrospective study. The electrophysiology laboratory and the pacemaker clinic databases were searched to retrieve all $\mathrm{AV}$ node ablation cases performed from 1994 to 2005 . The institution being a referal center, a large number of patients were sent for catheter ablation and had their follow-up remotely. Therefore, only cases with follow-up at our center were kept for analysis. Patients without left ventricular function study were excluded. The ejection fraction was obtained for the majority of patients by echocardiography $(>90 \%)$ and in rare cases by radionuclide study or left ventricular angiogram. Data was retreived by chart review by a single reviewer. An episode of decompensated heart failure was adjudicated when it was classified on the discharge summary as a primary or secondary diagnosis for any hospitalisation following the ablation procedure.

The ventricular pacing lead was placed at the right ventricular apical area. All patients had permanent pacemaker inserted prior to ablation. Atrio-ventricular node ablation was performed by radiofrequency application with a $4 \mathrm{~mm}$ tip catheter. The His was mapped and energy was delivered where the His deflection was recorded. Ablation parameters were set to a maximum of 100 watts and 60 degrees Celsuis. Radiofrequency was applied until atrioventricular dissociation and was then continued at least 60 to 120 seconds.

A total of seven hundred fifty-six patients (756) had AV node ablation. Five hundred fifty-two (552) had their followup remotely, and 119 patients of our institution had no follow-up echo and were not included. Fifteen patients had deceased and 4 patients with severe baseline left ventricular dysfunction awaiting heart transplant further underwent transplant and were excluded from the analysis. Five patients did not have successful AV node ablation and were ex- 
cluded. One hundred sixty-one charts (161) were included in the analysis. Table 1 displays baseline characteristics.

Table 1. Baseline Characteristics

\begin{tabular}{|l||c|}
\hline Age at Ablation (years) & $63 \pm 11$ \\
\hline Males (\%) & $77(48 \%)$ \\
\hline Chronic or persistant AF (\%) & $74(46 \%)$ \\
\hline Paroxysmal AF (\%) & $77(48 \%)$ \\
\hline Hypertension (\%) & $93(58 \%)$ \\
\hline Diabetes (\%) & $23(14 \%)$ \\
\hline Coronary artery disease (\%) & $66(41 \%)$ \\
\hline Non ischemic cardiomyopathy (\%) & $21(13 \%)$ \\
\hline Valvular heart disease (\%) & $44(27 \%)$ \\
\hline $\begin{array}{l}\text { Prior episode of decompensated heart failure } \\
\text { (no patients) }\end{array}$ & $34(21 \%)$ \\
\hline LVH (\%) & $62(38 \%)$ \\
\hline No heart disease & $55(34 \%)$ \\
\hline AF = Atrial Fibrillation; LVH = Left ventricular hypertrophy.
\end{tabular}

\section{STATISTICAL ANALYSIS}

Descriptive statistics are expressed as percentage and continuous variabled as means \pm standard deviation. Data was compared pre and post atrioventricular node ablation using paired-t-test when normaly distributed and Wilcoxon signed rank test for non parametric values. T-test for parametric and Wilcoxon signed rank test for non parametric values was used to compare data between groups. Normality test was automatically run by Sigmastat software for every statistical analysis. Nominal variables were compared with chi square or Fisher exact test when needed. A p value < 0.05 was considered significant. Multiple logistic regression test was used to test for confounding variables for categorical variables and multiple linear regression was used for continuous data.

\section{RESULTS}

One hundred sixty-one patients (161) were included in the analysis. Patients excluded because they had no followup LV function testing or had deceased were older than study patients, but other characteristics were similar: age $70 \pm 8$ (deceased) $67 \pm 12$ (excluded) vs $63 \pm 11$ years (study) (p $=0.01$ ), males $40 \%$ (deceased) vs $43 \%$ (excluded) vs $48 \%$ (study) ( $\mathrm{p}=0.65)$, PAF at baseline 75\% (deceased) vs 56\% (excluded) $v s 48 \%$ (study) $(\mathrm{p}=0.09)$. The ejection fraction of deceased patients was compared with study patients: pre ablation EF was $51 \pm 16 \%$ in the deceased population vs 53 $\pm 14 \%$ in the study patients $(\mathrm{p}=0.58)$. EF at last follow-up for deceased patients was $49 \pm 11 \%, p=0.95$ when compared to pre ablation EF in these same patients. The initial rhythm at the time of ablation was paroxsmal AF in $48 \%$ of patients and persistant or chronic AF in $46 \%$, and data was not available in 10 patients. One patient had AV node ablation for symptomatic hypertrophic cardiomyopathy treated by dual pacing. At follow-up, 17\% had paroxysmal AF and 77\% were in chronic $\mathrm{AF}$ and data was unavailable for 11 patients. Forty-seven patients (29\%) went from paroxysmal AF to chronic AF during the study period. Pacing modes are depicted in Table 2.

Table 2. Initial Pacing Mode Preceding Atrioventricular Junction Abation Compared to Pacing Mode at the Last Follow-Up

\begin{tabular}{|c|c|c|}
\hline & Pre-Ablation & Post-Ablation \\
\hline \hline VVI $(\%)$ & $67 \%(107)$ & $75 \%(119)$ \\
\hline DDD (\%) & $32 \%(51)$ & $23 \%(37)$ \\
\hline BiV (\%) & $0.6 \%(1)$ & $2 \%(3)$ \\
\hline P>0.1 Missing data for 2 patients.
\end{tabular}

The mean clinical follow-up was $69 \pm 38$ months (median 66 months) and the follow-up left ventricule function study was done $55 \pm 38$ months post ablation (median 50 months). Left ventricular ejection fraction prior to the atrioventricular junction ablation was $53 \pm 14 \%$ and decreased to $51 \pm 13 \%$ at follow-up ( $<<0.05$ ). No significant change was observed in left atrial and left ventricular sizes, and in the severity of mitral regurgitation. The echocardiographic parameters are shown in Table 3. When pre and post ablation EF was compared in the whole cohort, 55 patients lowered their ejection fraction (EF) by $5 \%$ or more. Of that group, 47 patients had an initial EF of $50 \%$ or more. Twenty-nine percent raised their EF by $5 \%$ or more. In the subgroup of patients with low baseline $\mathrm{EF}(\leq 40 \%), 1.5 \%$ lowered their EF by $5 \%$ or more and $14 \%$ raised their EF by $5 \%$ or more at follow-up. The EF over time categorized in normal $(\geq 50 \%)$, mild $(40-49 \%)$, moderate $(30-39 \%)$ and severe $(<30 \%)$ ventricular dysfunction is illustrated in Fig. (1). In the group of patients with a baseline $\mathrm{EF} \geq 50 \%, 8 \%$ developed a moderate LV dysfunction with an $\mathrm{EF} \leq 40 \%$ at their last follow-up. Five percent of patients with a baseline moderate LV dysfunction normalized their EF at follow-up.

Table 3. Echocardiographic and Heart Function Study Parameters

\begin{tabular}{|c|c|c|c|}
\hline & Pre-Ablation & Post-Ablation & p \\
\hline \hline LVED diameter (mm) & $49.5 \pm 5.8$ & $50.5 \pm 7.4$ & 0.98 \\
\hline LA diameter (mm) & $46.4 \pm 10.3$ & $49.0 \pm 8.7$ & 0.14 \\
\hline Ejection fraction (\%) & $\mathbf{5 3 . 4} \pm \mathbf{1 4 . 4}$ & $\mathbf{5 0 . 8} \pm \mathbf{1 2 . 7}$ & $\mathbf{0 . 0 2}$ \\
\hline Mitral Regurgitation* & $1.37 \pm 1.08$ & $1.36 \pm 1.13$ & 0.92 \\
\hline
\end{tabular}

LVED: Left ventricular end diastolic; LA: Left atrium.

* Mitral Regurgitation graded from 1 to 4, according to the American Society of Echocardiography recommendations [10].

Thirty-one patients suffered at least one episode of decompensated heart failure post ablation. Of those 31 patients, only 1 had no other associated conditions that could contribute to or cause the decompensation. Moreover, among the 31 patients who suffered decompensated heart failure, one presented with acute pulmonary edema and hypertensive crisis, and 2 others had severe obstructive valvulopathy that lead to valve repair or replacement. In a multiple logistic regression model, high blood pressure, ischemic heart disease and valvular heart disease were significant and independant predictors of heart failure episodes during follow-up. Baseline 


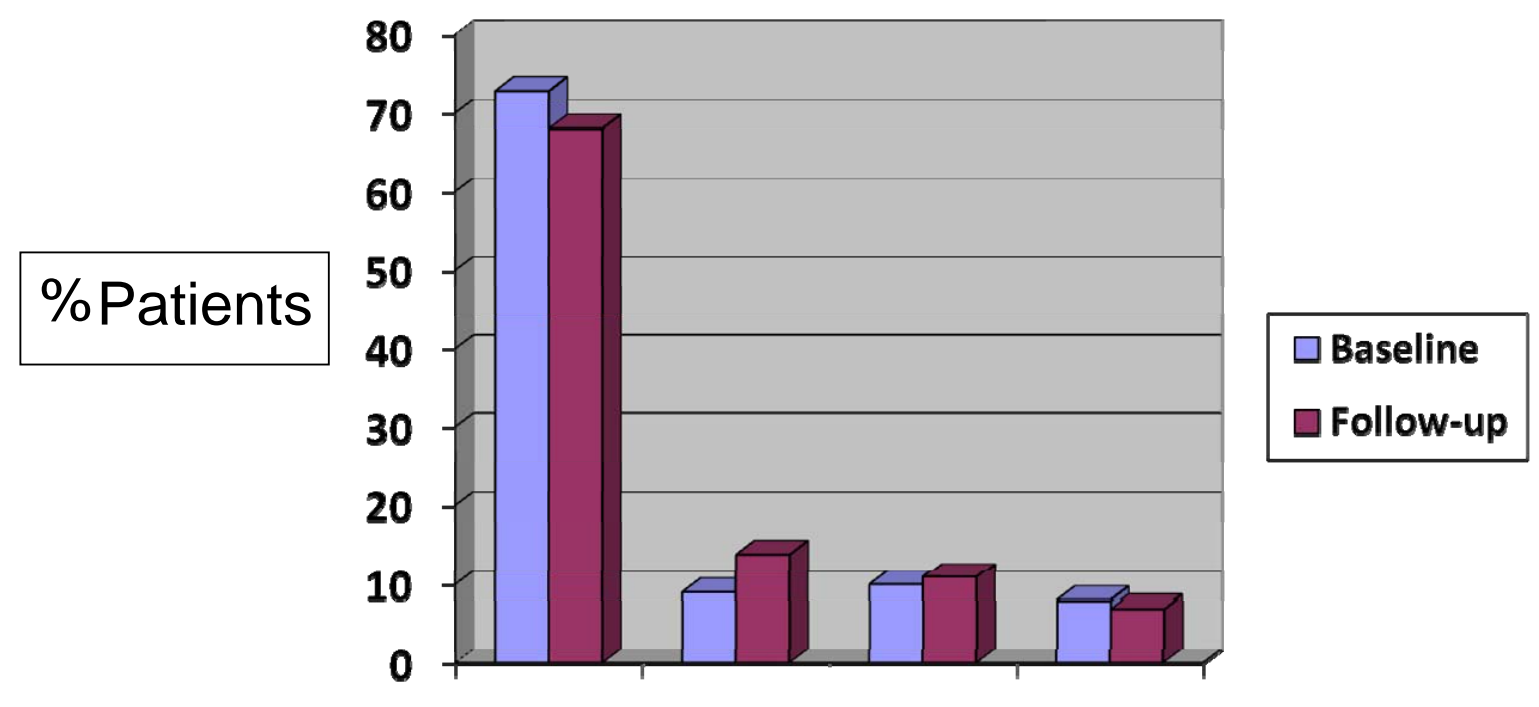

\section{$E F \geq 50 \%$ EF $40-49 \%$ EF $30-39 \%$ EF $<30 \%$}

$\mathrm{p}>0.05$

EF: Ejection fraction

Fig. (1). Distribution of left ventricular ejection fraction of the study population at baseline and at follow-up.

ejection fraction was also an independant predictor of heart failure at follow up $(\mathrm{p}<0.001)$. Table 4 lists the co-morbid conditions and their potential contribution in patients with heart failure episodes.

Table 4. Heart Failure and Associated Conditions

\begin{tabular}{|l|c|c|c|}
\hline \multicolumn{1}{|c|}{$\begin{array}{c}\text { Associated } \\
\text { Conditions }\end{array}$} & $\begin{array}{c}\text { Number of } \\
\text { Patients }\end{array}$ & $\begin{array}{c}\text { Odds Ratio } \\
\text { (CI) }\end{array}$ & P \\
\hline \hline Total population with HF & 31 & & \\
\hline High blood pressure & 24 & 9.1 & $<0.001$ \\
\hline Coronary heart disease & 19 & 3.6 & 0.01 \\
\hline Valvular heart disease & 8 & 3.7 & 0.02 \\
\hline Dilated cardiomyopathy & 4 & NS & NS \\
\hline Diabetes & 3 & NS & NS \\
\hline Baseline VVI pacing & 20 & NS & NS \\
\hline No associated conditions & 1 & NS & NS \\
\hline
\end{tabular}

$\mathrm{EF}=$ Ejection fraction; $\mathrm{HF}=$ Heart Failure; $\mathrm{CI}=$ Confidence interval; NS = Non significant.

Three patients developped severe (4/4) mitral regurgitation during follow-up and all had previous moderate (3/4) mitral regurgitation [10]. Eight patients with mild to moderate mitral regurgitation developped moderate regurgitation at follow-up.

Subgroup analysis was perfomed to compare patients with baseline paroxysmal atrial fibrillation who stayed in paroxysmal AF to patients who developed chronic AF. Patients who evolved to chronic AF had a higher baseline degree of mitral regurgitation $(1.6$ vs $0.7, \mathrm{p}=0.02)$ and were more frequently paced in a VVI mode $(71 \%$ vs $21 \%$, $\mathrm{p}<0.001)$. At follow-up, patients in chronic AF had a larger LA (48 vs $45 \mathrm{~mm}, \mathrm{p}=0.04$ ) (Table 5). A second subgroup
Table 5. Subgroups: Paroxysmal Atrial Fibrillation; Maintenance of Paroxysmal AF Versus Evolution to Chronic AF

\begin{tabular}{|l|c|c|c|}
\hline & $\begin{array}{c}\text { PAF } \rightarrow \\
\text { PAF [47] }\end{array}$ & $\begin{array}{c}\text { PAF } \rightarrow \\
\text { CAF [30] }\end{array}$ & P \\
\hline \hline Age (years) & $62 \pm 10$ & $62 \pm 12$ & 0.87 \\
\hline Males (\%) & $42 \%$ & $44 \%$ & 0.99 \\
\hline High Blood Pressure (\%) & $44 \%$ & $62 \%$ & 0.15 \\
\hline Coronary Artery Disease (\%) & $44 \%$ & $28 \%$ & 0.20 \\
\hline Valvular heart disease (\%) & $18 \%$ & $26 \%$ & 0.49 \\
\hline Other heart disease (\%) & $12 \%$ & $17 \%$ & 0.69 \\
\hline HF prior to ablation (\%) & $29 \%$ & $43 \%$ & 0.28 \\
\hline Baseline EF (\%) & $55 \pm 17$ & $55 \pm 13$ & 0.70 \\
\hline Baseline LVEDD (mm) & $50 \pm 5$ & $49 \pm 6$ & 0.44 \\
\hline Baseline LA size(mm) & $42 \pm 10$ & $46 \pm 13$ & 0.31 \\
\hline Baseline mitral regurgitation & $\mathbf{0 . 7} \pm \mathbf{0 . 8}$ & $\mathbf{1 . 6} \pm \mathbf{1 . 0}$ & $\mathbf{0 . 0 2}$ \\
\hline Baseline VVI (\%) & $\mathbf{2 1 \%}$ & $\mathbf{7 0 \%}$ & $<\mathbf{0 . 0 0 1}$ \\
\hline Follow-up EF (\%) & $50 \pm 13$ & $54 \pm 12$ & 0.11 \\
\hline Follow-up LVEDD (mm) & $51 \pm 8$ & $49 \pm 7$ & 0.31 \\
\hline Follow-up LA size (mm) & $\mathbf{4 5 \pm 1 0}$ & $\mathbf{4 8} \pm \mathbf{8}$ & $\mathbf{0 . 0 4}$ \\
\hline Follow-up mitral regurgitation & $1.0 \pm 1.0$ & $1.4 \pm 1.1$ & 0.18 \\
\hline Follow-up VVI (\%) & $\mathbf{2 4 \%}$ & $\mathbf{8 5 \%}$ & $<\mathbf{0 . 0 0 1}$ \\
\hline HF episode follow-up (\%) & $24 \%$ & $21 \%$ & 0.97 \\
\hline
\end{tabular}

$\mathrm{HF}=$ Heart Failure $; \mathrm{EF}=$ Ejection fraction; LVEDD = Left ventricular end-diastolic diameter; $\mathrm{LA}=\mathrm{Left}$ atrium; $\mathrm{PAF}=$ Paroxysmal atrial fibrillation $\mathrm{CAF}=\mathrm{Chronic}$ atrial fibrillation. Mitral regurgitation is expressed as a continuous value and is graded from 1 to 4 according to the recommendations of the American Society of Echocardiography [10]. 
analysis was done to compare baseline lone AF to paroxysmal AF and to chronic AF. Patients with lone AF were younger, had smaller baseline and follow-up LA size and were more frequently paced in a DDD mode. Patients with baseline PAF had significantly less coronary heart disease and were more frequently paced in dual mode (DDD) compared to patients with chronic AF. Other characteristics did not differ in a statistically significant way (Table 6).

Table 6. Lone AF Versus Paroxysmal AF Versus Chronic AF

\begin{tabular}{|c|c|c|c|c|}
\hline & $\begin{array}{c}\text { Lone } \\
\text { AF } \\
{[21]}\end{array}$ & $\begin{array}{c}\text { Baseline } \\
\text { PAF } \\
\text { [77] }\end{array}$ & $\begin{array}{c}\text { Baseline } \\
\text { CAF } \\
\text { [74] }\end{array}$ & $\mathbf{P}$ \\
\hline Age (years) & $56 \pm 13$ & $62 \pm 12$ & $65 \pm 11$ & $0.005^{1}$ \\
\hline Males $(\%)$ & $57 \%$ & $43 \%$ & $54 \%$ & 0.25 \\
\hline High Blood Pressure (\%) & 0 & $36 \%$ & $47 \%$ & $0.18^{3}$ \\
\hline Coronary Artery Disease (\%) & 0 & $14 \%$ & $42 \%$ & $<0.001^{3}$ \\
\hline Valvular heart disease $(\%)$ & 0 & $23 \%$ & $34 \%$ & $0.18^{3}$ \\
\hline Other heart disease $(\%)$ & 0 & $15 \%$ & $8 \%$ & $0.27^{3}$ \\
\hline HF prior to ablation (\%) & 0 & $39 \%$ & $46 \%$ & $0.47^{3}$ \\
\hline Baseline EF (\%) & $60 \pm 6$ & $55 \pm 15$ & $52 \pm 14$ & 0.19 \\
\hline Baseline LVEDD (mm) & $49 \pm 7$ & $49 \pm 5$ & $50 \pm 6$ & 0.85 \\
\hline Baseline LA size (mm) & $36 \pm 5$ & $45 \pm 11$ & $48 \pm 9$ & $0.01^{1}$ \\
\hline Baseline mitral regurgitation & $0.8 \pm 0.5$ & $1.3 \pm 1.0$ & $1.4 \pm 1.1$ & 0.49 \\
\hline Baseline VVI (\%) & $52 \%$ & $49 \%$ & $88 \%$ & $<0.001$ \\
\hline Follow-up EF (\%) & $55 \pm 8$ & $52 \pm 12$ & $49 \pm 13$ & 0.17 \\
\hline Follow-up LVEDD (mm) & $51 \pm 77$ & $50 \pm 7$ & $51 \pm 8$ & 0.57 \\
\hline Follow-up LA size (mm) & $44 \pm 7$ & $47 \pm 9$ & $50 \pm 8$ & $0.02^{2}$ \\
\hline Follow-up mitral regurgitation & $1.2 \pm 0.8$ & $1.2 \pm 1.1$ & $1.2 \pm 1.0$ & 0.99 \\
\hline Follow-up VVI (\%) & $57 \%$ & $60 \%$ & $92 \%$ & $<0.001$ \\
\hline HF at follow-up (\%) & $5 \%$ & $25 \%$ & $18 \%$ & 0.11 \\
\hline \multicolumn{5}{|c|}{$\begin{array}{l}\mathrm{HF}=\text { Heart Failure; } \mathrm{EF}=\text { Ejection fraction; } \mathrm{LVEDD}=\mathrm{Left} \text { ventricular end-diastolic } \\
\text { diameter; } \mathrm{LA}=\mathrm{Left} \text { atrium; } \mathrm{MR}=\text { Mitral regurgitation; PAF }=\text { Paroxysmal atrial } \\
\text { fibrillation; } \mathrm{CAF}=\text { Chronic atrial fibrillation. Mitral regurgitation is expressed as a } \\
\text { continuous value and is graded from } 1 \text { to } 4 \text { according to the recommendations of the } \\
\text { American Society of Echocardiography (10). } \\
{ }^{1} \text { Lone AF statistically different from PAF and CAF. } \\
{ }^{2} \text { Lone AF statistically different from CAF. } \\
{ }^{3} \text { Comparing CAF and PAF only. }\end{array}$} \\
\hline
\end{tabular}

\section{DISCUSSION}

Atrioventricular node ablation followed with right ventricular apical pacing was performed for the past 15 years at our institution, and is well tolerated by the majority of patients. This study is one with the longest follow-up of such a cohort, with a mean follow-up of more than 5 years. A recent study with a 4.3 years follow-up of a similar population reported no change in functionnal class, an improvement in left ventricular fractional shortening, a significant decrease in left ventricular end diastolic diameter and $20 \%$ hospitalisation for heart failure [11]. Others have reported no deleterious effect on left ventricular function [12]. In the present study, a mild decrease in ejection fraction and an increment in left atrial diameter is noted in the total population. The ventricular dyssynchrony induced by right ventricular apical pacing could partially explain this deterioration. The fact that most of the changes in EF occur in patients with normal baseline EF whom at follow-up developed mild left ventricular dysfunction, as depicted in Fig. (1), supports this hypothesis. Only 8\% of patients with normal left ventricular systolic function developed moderate heart failure as defined by an ejection fraction below $40 \%$. Moreover, only 2 patients necessitated upgrade of their pacing device for a resychronization system. In a previous meta analysis, an improvement of $4.4 \%$ in the $\mathrm{EF}$ was reported ${ }^{3}$. Others have also reported improvement in left ventricular function [13]. In our study, a lowering of EF could be explained by a higher baseline $\mathrm{EF}(53 \%)$ in our population compared to the cohorts represented in the meta analysis (EF: $27-51 \%$ ). In patients with baseline moderate heart failure, $5 \%$ normalized their ejection fraction at follow-up. This subgroup probably represents a population with reversible tachycardiomyopathy secondary to a rapid ventricular response rate in AF. Few patients developed or aggravated mitral regurgitation.

In our study, $29 \%$ of paroxysmal AF became chronic AF, and at last follow-up, $82 \%$ of patients were in chronic AF. In a previous study by Gillis and al, the rate of chronic AF after one year of follow-up was $42 \%$ [14]. The higher incidence of permanent $\mathrm{AF}$ following $\mathrm{AV}$ node ablation in the present study can be secondary to the longer follow-up, as the rate of permanent AF may increase over time as a result of electrical remodeling of the atria.

In previous studies, $\mathrm{AV}$ node ablation followed by right ventricular pacing has proven safe and beneficial for patients. Survival was not affected significantly [4] and quality of life and exercice capacity was improved in treated patients $[3,15]$. This study was not designed nor powered to retreive mortality data, but demonstrated that the magnitude of heart function deterioration was low (mean EF lowered by $2 \%$ ) since only 2 patients developed systolic dysfunction leading to an upgrade to biventricular pacing. Moreover, there was no significant heart chamber enlargement in the overall population. Although several patients had episodes of decompensated heart failure, most had co-existing conditions that could cause or contribute to their clinical deterioration. As expected, concurrent cardiovascular diseases and lower baseline ejection fraction were predictors of subsequent episodes of heart failure and it is interesting to note only one patient with lone AF at baseline experienced heart failure at follow-up.

As emerging therapies such as atrial fibrillation ablation and biventricular pacing are becoming more frequently performed, and are evolving toward being standards of care for specific populations, it is justified to revisit the role of the ablate and pace strategy in the therapeutic algorithm of atrial fibrillation management. According to the 2006 American Heart Association / American College of Cardiology /European Society of Cardiolgy guidelines [16], it is a valuable strategy in chosen patients. The PAVE study compared VVIR and biventricular (BiV) pacing post $\mathrm{AV}$ node ablation and concluded that walking distance was improved with BiV pacing, left ventricular ejection fraction stayed stable with BiV pacing and lowered with VVIR, but no difference in survival or quality of life scores was detected [17]. It is therefore not necessary to initiate biventricular pacing upfront following AV node ablation. When compared with atrial fibrillation ablation, AV node ablation with pacing resulted in less sympto- 
matic arrhythmias than AF ablation, but more patients with $\mathrm{AV}$ node ablation and pacing developped heart failure [18].

\section{LIMITATIONS}

This study is a retrospective analysis of a subgroup of patients from our total AV node ablation population. It is a single center study including a non-consecutive serie of patients. The analysed data is observational and cannot be used to establish cause-effect relationship thus the underlying mechanisms to explain heart function modification and clinical events are hypothetical. Many patients have not been followed at our institution and may have developed heart failure over time but were not collected in our database. Of note, since we are the only tertiary cardiology centre implanting resynchronization devices in the area, any upgrade to biventricular pacing would likely have been collected in our database. Since clinical events, ejection fraction and echocardiographic parameters were collected retrospectively, no standard protocol for measurements or assessment was used.

\section{CONCLUSION}

$\mathrm{AV}$ node ablation and right ventricular pacing remain a therapeutic option in selected patients, mostly those with uncontrolled atrial fibrillation who are not candidate or decline primary atrial fibrillation ablation. It is associated with a minimal but significant degree of systolic dysfunction, but the clinical impact is probably small for most patients. Biventricular pacing should be reserved for patients who remain in heart failure or develop heart failure despite rate control and medical therapy, for those with heart failure not due to tachycardiomyopathy or for patients with baseline moderate to severe mitral regurgitation. In times where atrial fibrillation can be cured, sinus rhythm can be restored and AV conduction can be preserved, the ablate and pace stragegy still has a place in the treatment of atrial fibrillation but only after consideration of all other options. Even if AV node ablation and pacing is safe, we have to keep in mind that it is a palliative and irreversible procedure. In young patients, the risk of long term pacing complications, infections, lead dislogement and lead fracture, is increased by the higher number of procedure that will be necessary over a lifespan.

\section{ABBREVIATIONS}

$$
\begin{array}{ll}
\mathrm{AF} & =\text { Atrial Fibrillation } \\
\mathrm{AV} \text { node } & =\text { Atrioventricular node } \\
\mathrm{EF} & =\text { Ejection fraction } \\
\mathrm{LV} & =\text { Left ventricule }
\end{array}
$$

\section{REFERENCES}

[1] The AFFIRM investigators, A comparison of rate control and rhythm in patients with atrial fibrillation. N Engl J Med 2002; 347: 1825-1833.
[2] Cappato R, Calkins H, Chen SA, et al. Worldwide survey on the methods, efficacy and safety of catheter ablation for human atrial fibrillation. Circulation 2005; 111: 1100-1105.

[3] Wood MA, Brown-Mahoney C, Kay NG, Ellenbogen KA. Clinical outcomes after ablation and pacing therapy for atrial fibrillation. A meta-analysis. Circulation 2000; 101: 1138-44.

[4] Ozcan C, Jahangir A, Friedman PA, et al. Long-term surival after ablation of the atrioventricular node and implantation of a permanent pacemaker in patients with atrial fibrillation. $\mathrm{N}$ Engl $\mathrm{J}$ Med 2001; 344: 1043-1051.

[5] Ozcan C, Jahangir A, Friedman PA, et al. Significant effects of atrioventricular node ablation and pacemaker implatation on left ventricular function and long-term survival in patients with atrial fibrillation and left ventricular dysfunction. Am J Cardiol 2003; 92: 33-37.

[6] Geelen P, Brugada J, Andries E, Brugada P. Ventricular Fibrillation and Sudden Death After Radiofrequency Catheter Ablation of the Atrioventricular Junction. Pacing Clin Electrophysiol 1997, 20: 343-348.

[7] Tops LF, Schalij MJ, Holman ER, Van Erven L, Van der Wall EE, Bax JJ. Right ventricular pacing can induce ventricular dyssynchrony in patients with atrial fibrillation after AV node ablation. J Am Coll Cardiol 2006; 48: 1642-1648.

[8] Hayes JJ, Sharma AD, Love JC, Herre JM, Leonen AO, Kudenchuk PJ for the DAVID investigators. Abnormal conduction increases risks of adverse outcomes from right ventricular pacing. J Am Coll Cardiol 2006; 48: 1628-33.

[9] Manolis A. The deleterious consequences of right ventricular, apical pacing: Time to seek alernate site pacing. PACE 2006; 29: 298-315.

[10] Zoghbi WA, Enriquez-Sarano M, Foster E, et al. American Society of Echocardiography. Recommendations for evaluation of the severity of native valvular regurgitation with two-dimensional and Doppler echocardiography. J Am Soc Echocardiogr 2003; 16: 777802.

[11] Tan ES, Rienstra M, Wiesfeld ACP, Schoonderwoerd BA, Hobbel HHF, Van Gelder IC. Long-term outcome of the atrioventricular node ablation and pacemaker implantation for symptomatic refractory atrial fibrillation. Europace 2008; 10: 412-418.

[12] Chen L, Hodge D, Jahangir A, et al. Preserved left ventricular ejection fraction following atrioventricular junction ablation and pacing for atrial fibrillation. J Cardiovasc Electrophysiol 2008; 19:19-27.

[13] Kay GN, Ellenbogen KA, Giudici M, et al. The Ablate and Pace Trial: a prospective study of catheter ablation of the AV conduction system and permanent pacemaker implantation for treatment of atrial fibrillation. APT Investigators. J Interv Card Electrophysiol 1998; 2:121-35.

[14] Gillis AM. Selection of pacing mode after interruption of atrioventricular conduction for atrial fibrillation: observations from the PA3 clinical trial. Card Electrophysiol Rev 2003; 7: 312-314.

[15] Kay GN, Bubien RS, Epstein AE, Plumb VJ. Effect of catheter ablation of the atrioventricular junction on quality of life and exercice tolerance in paroxysmal atrial fibrillation. Am J Cardiol 1988; 62: 741-744.

[16] European Heart Rhythm Association and the Heart Rhythm Society, Valentin F, Lars ER, David SC, et al. Guidelines for the Management of Patients With Atrial Fibrillation). J Am Coll Cardiol 2006; 48: 854-906.

[17] Doshi RN, Daoud EG, Fellows C, et al. For the PAVE study group. Left ventricular-based cardiac stimulation post AV nodal ablation evaluation (The PAVE study). J Cardiovasc Electrophysiol 2005; 16: 1160-65.

[18] Hseih MH, Tai CT, Lee SH, et al. Catheter ablation of atrial fibrillation versus atrioventricular junction ablation plus pacing therapy for elderly patients with medically refractory paroxysmal atrial fibrillation. J Cardiovasc Electrophysiol 2005; 16: 457-461. 